\section{RMD Open}

Rheumatic \&

Musculoskeletal Diseases

To cite: Kragstrup TW, Glintborg B, Svensson AL, et al. Waiting for JAK inhibitor safety data. RMD Open 2022;8:e002236. doi:10.1136/ rmdopen-2022-002236

Received 24 January 2022 Accepted 4 February 2022

\section{Check for updates}

C) Author(s) (or their employer(s)) 2022. Re-use permitted under CC BY-NC. No commercial re-use. See rights and permissions. Published by BMJ.

For numbered affiliations see end of article.

Correspondence to Dr Tue Wenzel Kragstrup; kragstrup@biomed.au.dk

Dr David FL Liew; david.liew@austin.org.au

\title{
Waiting for JAK inhibitor safety data
}

\author{
Tue Wenzel Kragstrup (D) , ,2 Bente Glintborg (D) ,3,4 Annemarie L Svensson, ${ }^{3}$ \\ Christopher McMaster, ${ }^{6,7,8}$ Philip C Robinson (D) , 9,10 Bent Deleuran (D) ,1,11 \\ David FL Liew (1) $6,7,12$
}

\section{ABSTRACT}

The US Food and Drug Administration (FDA) has recently added a new 'black box warning' on all currently approved Janus kinase (JAK) inhibitors indicated for the treatment of arthritis and other inflammatory conditions based on results from the ORAL Surveillance study of tofacitinib versus tumour necrosis factor alpha inhibitors in rheumatoid arthritis. This is a warning difficult to ignore because the data, being from a randomised controlled trial, are of high fidelity and hard to reproach. It is especially problematic because safety data for all the other JAK inhibitors will be pending for several years. So how might we proceed, without being bound by our stasis? The lack of absolute certainty seems to require a pragmatic approach to the routine care use of JAK inhibitors. The patients who were at greatest risk were older and had other risk factors for the corresponding adverse events, in keeping with effect modification. This highlights the need to focus on risk stratification when tailoring therapy. In this viewpoint, we propose a simple illustration to guide clinical decision-making. First, identify general risk factors for venous thromboembolic event (VTE), major adverse cardiac event (MACE) and cancer (age>65 years and smoking) and whether there is a previous history of VTE, MACE or cancer. Then, evaluate risk based on the number of other risk factors for VTE and the number of other risk factors for MACE. Ultimately, 'treat-to-target' will in the end always be 'treat-to-agreement'. As we have done in the past, and will do in the future, the optimal treatment strategy will have to be tailored based on individual patient risk factors and preferences in a shared-decision process.

\section{NEW US FOOD AND DRUG ADMINISTRATION WARNINGS FOR JANUS KINASE INHIBITORS}

'Let us go. We cannot. Why not? We are waiting for Godot.' This quote is from the tragicomedy Waiting for Godot by Samuel Beckett. "Do not let us do anything, it is safer", says one of the characters. In the play, the lack of necessary information makes the characters completely helpless; they are stuck in the same place. In an analogous way, rheumatologists are waiting for more safety data for the Janus kinase (JAK) inhibitors. The US Food and Drug Administration (FDA) has recently added a new 'black box warning' on all currently approved JAK inhibitors indicated for the treatment of arthritis and other inflammatory conditions including tofacitinib, baricitinib and upadacitinib. More precisely, the FDA added warnings regarding an 'increased risk of serious heart-related events, cancer, blood clots, and death for JAK inhibitors that treat certain chronic inflammatory conditions'. The warning was based on the ORAL Surveillance study on tofacitinib now included in the Xeljanz European Medicine Agency (EMA) Summary of Product Characteristics (SmPC) ${ }^{2}$ and the FDA Prescribing Information. ${ }^{3}$ The currently approved JAK inhibitors have differences in preferential, dose-dependent specificity for the four JAK enzymes: JAK1, JAK2, JAK3 and TYK2. However, the mechanism for the reported adverse events is not understood and it is currently not known whether JAK specificity alters the safety profile. ${ }^{45}$ The FDA warning has therefore been applied to all JAK inhibitors approved in the USA (tofacitinib, baricitinib and upadacitinib) with a recent update stating that "these medicines (should be reserved) for patients who have had an inadequate response or intolerance to one or more TNF blockers'. ${ }^{6}$ This is a warning difficult to ignore, not just because of the extrapolation of data from tofacitinib to all JAK inhibitors, but also because the data, being from a randomised controlled trial, are of high fidelity and, despite some limitations, harder to reproach.

\section{THE ORAL SURVEILLANCE STUDY: A POST- MARKETING REQUIREMENT, CHALLENGING CURRENT PRACTICE}

ORAL Surveillance was a prospective, phase $3 \mathrm{~b} / 4$, randomised, open-label, noninferiority, safety endpoint study comparing tofacitinib and tumour necrosis factor (TNF) inhibitors, mandated as part of postmarketing requirements and commitments to the FDA (NCT02092467). Patients included had rheumatoid arthritis (RA) and an inadequate response to methotrexate. At time of inclusion, patients should be at least 50 years old and were mandated to have at least one 
additional cardiovascular (CV) risk factor (current cigarette smoking, hypertension, hypercholesterolaemia, diabetes mellitus, family history of premature coronary heart disease, extra-articular disease associated with RA, history of coronary artery disease). The incidence rates from the ORAL Surveillance study about the risk of venous thromboembolic events (VTEs), ${ }^{7}$ major adverse cardiac events (MACEs) ${ }^{8}$ and malignancies ${ }^{9}$ was recently presented at the ACR Convergence and the first publication from the study was recently published. ${ }^{10}$ Two years earlier, in 2019, tofacitinib $10 \mathrm{mg}$ two times per day was shown to increase the risk of VTE, and proper warnings were given in a Pfizer press release ${ }^{11}$ — with $5 \mathrm{mg}$ two times per day subsequently being the only dose approved by the FDA and EMA for adult patients with RA and psoriatic arthritis. The relevant question from the ORAL Surveillance study is therefore whether safety outcomes differ between the $5 \mathrm{mg}$ twice daily dose versus TNF inhibitors. Incidence rates for VTE were numerically higher for tofacitinib $5 \mathrm{mg}$ two times per day compared with TNF inhibition. ${ }^{7}$ In both treatment groups, risk factors for pulmonary embolism were age, male sex, body mass index (BMI) $\geq 30 \mathrm{~kg} / \mathrm{m}^{2}$, history of hypertension or VTE, and use of oral contraceptives or hormone replacement therapy, corticosteroids or antidepressants. Further, the tofacitinib $5 \mathrm{mg}$ twice daily dose was associated with a numerically higher incidence of MACE compared with TNF inhibitor treatment especially among patients aged $\geq 65$ years, those who had ever smoked and aspirin users. ${ }^{8}$ MACE mostly occurred in patients with a history of coronary artery disease) or risk factors of MACE. Tofacitinib $5 \mathrm{mg}$ two times per day was also associated with all malignancies excluding non-melanoma skin cancer (NMSC). ${ }^{9}$ Incidence rates were numerically higher in current and past smokers and patients aged $\geq 65$ years. Lung cancer was the most frequently reported cancer type in patients treated with tofacitinib (after excluding NMSC). The conclusion of the ORAL Surveillance study was that tofacitinib was not non-inferior to TNF inhibitors. However, as stated by the authors, the study was not powered for comparisons of individual tofacitinib dose groups with TNF inhibitor for any of the safety outcomes. ${ }^{7-10}$ Indeed, while executing the perfect trial is always limited by financial and practical circumstances, further challenges from the trial design could be added: the study was open label, there were geographical differences in the TNF inhibitor used, and the tofacitinib $10 \mathrm{mg}$ twice daily dose was withdrawn during the study period. ${ }^{10}$ Despite these questions, the concern surrounding JAK inhibitors remains more robust than it has ever been.

\section{SPARSITY OF DATA}

The mechanisms behind the potential risk for VTE, MACE and cancer associated with JAK inhibition are still inadequately explained, despite admirable attempts to do so. ${ }^{12}$ It is important to note that meta-analyses of the clinical trial programmes of tofacitinib, baricitinib, upadacitinib and filgotinib did not reveal a significant risk for VTE, MACE or cancer. ${ }^{13-16}$ However, the data from ORAL Surveillance indicate that safety analysis from the clinical development programmes, with their carefully selected patient population, might not capture the realworld risk for VTE, MACE and cancer in patients with increased cardiovascular risk at treatment start. Therefore, it is problematic that safety data for all the other JAK inhibitors will be pending for several years. The post-marketing safety study mandated by the FDA for baricitinib is planned to finish in 2026 (NCT03915964). Further, several cohort studies are listed in the European Network of Centres for Pharmacoepidemiology and Pharmacovigilance (ENCePP) website $^{17}$ as ongoing or planned (EUPAS25151, EUPAS25145, EUPAS25142, EUPAS32271, EUPAS34213, EUPAS25139 and EUPAS25164). Post-marketing randomised studies for upadacitinib and filgotinib have not yet been mandated by the FDA (filgotinib is not approved by FDA). AbbVie has a 5-year extension phase on the SELECT-COMPARE study of upadacitinib (NCT02629159) and both European and US-based post-marketing surveillance programmes (EUPAS39217, EUPAS39194, EUPAS39211). Similarly, post-marketing surveillance is ongoing by Galapagos NV for filgotinib (eg, EUPAS39767). Compared with the pre-biological disease-modifying antirheumatic drug (DMARD) era, where we urgently needed means for morbidity-reducing disease control, we now have a luxury situation where multiple highly effective options can be tailored to patients, balancing all considerations with their pros and cons. In weighing up our options, what risks should we advise of?

\section{BALANCING THE RISKS}

Let us assume the results about risk of VTE, MACE and cancer of ORAL Surveillance are accurate. Then we can calculate the number of subjects with event/10000 person-years. The incidence rate of VTE was 33/10000 person-years for tofacitinib $5 \mathrm{mg}$ two times per day and 20/10000 person-years for TNF inhibitor. The incidence rate of MACE was 91/10000 person-years for tofacitinib and 73/10000 person-years for TNF inhibitor. The incidence of myocardial infarction was numerically higher with tofacitinib compared with TNF inhibitor, while the incidence of stroke was similar across the treatment groups. The incidence of cancer was numerically higher with tofacitinib compared with TNF inhibitor. For lung cancer, the numbers were 23/10000 person-years and $13 / 10000$ person-years, respectively. Notably, the incidence of lung cancer was close to three times higher in the USA compared with the rest of the world. So, does this small increase in risk change our risk-benefit assessment of JAK inhibitors? For example, the HR of coronary heart disease with smoking can be used for perspectivation. To provide some comparison, in a prospective population-based study from the USA, the HR compared 
with non-smokers of coronary heart disease in men who smoke 20 cigarettes or more a day was 2.90 (5.83 for women). ${ }^{18}$ The HR of myocardial infarction in ORAL Surveillance was 1.69 for tofacitinib $5 \mathrm{mg}$ two times per day compared with TNF inhibitors. It is also important to acknowledge that inflammatory disease activity itself is associated with increased risk of VTE, ${ }^{19} \mathrm{MACE}^{20}$ and cancer. ${ }^{21}$ Therefore, doing nothing is certainly not an option. When the benefits from a JAK inhibitor are significant, for example, oral dosing in needle phobia, issues with intermittent dosing or loss of efficacy from antidrug antibodies, then a pragmatic assessment not just of relative risk increases but absolute risk is needed. In this respect, it is also important to acknowledge that the risk with drugs with other modes of action have not been elucidated including methotrexate, IL-6 receptor inhibitors, abatacept and rituximab. For example, in a recent US Medicare claims study with a large real-world cohort of older patients with RA, treatment with methotrexate was associated with a twofold increased risk of VTE relative to hydroxychloroquine. ${ }^{22}$ Dose may also become a consideration, particularly given the circumstance that variable doses for both baricitinib (2 and $4 \mathrm{mg}$ ) and filgotinib (100 and $200 \mathrm{mg}$ ) are licensed in Europe. So how might we proceed, without being bound by our stasis? The lack of absolute certainty seems to require a pragmatic approach to the routine care use of JAK inhibitors. Even within the population enriched for cardiovascular risk factors included in the ORAL Surveillance study, the patients who were at greatest risk were older and had other risk factors for the corresponding adverse events, in keeping with effect modification. This is in line with a recent study of three US claims databases where tofacitinib was associated with an increased risk of cardiovascular outcomes (statistically non-significant) only in patients with cardiovascular risk factors. ${ }^{23}$

This highlights the need to focus on risk stratification when tailoring therapy, as we propose in the illustration (figure 1). Rheumatologists are not used to considering risk of MACE in the context of modern DMARD standard of care in RA, whereas this assessment is customary when prescribing non-steroidal anti-inflammatory drugs (NSAIDs), which may be forgotten in RA but are still vital throughout rheumatology. Such risk assessment comprises history of previous MACE and cardiovascular risk factors including hypertension, hypercholesterolaemia and diabetes, and the relative risk-benefit ratio of different agents within the broader class is considered. Where no alternative exists, such as in first-line therapy of axial spondyloarthritis, risk is tolerated and mitigated wherever possible-by managing the underlying cardiovascular risk, through the management of comorbidities, by a shared decision-making process which addresses all modifiable risk factors including smoking and obesity in a holistic approach to disease that extends beyond the prescription pad. Although there are differences in its place in the treatment algorithms, the NSAID approach seems a familiar paradigm extrapolatable to JAK

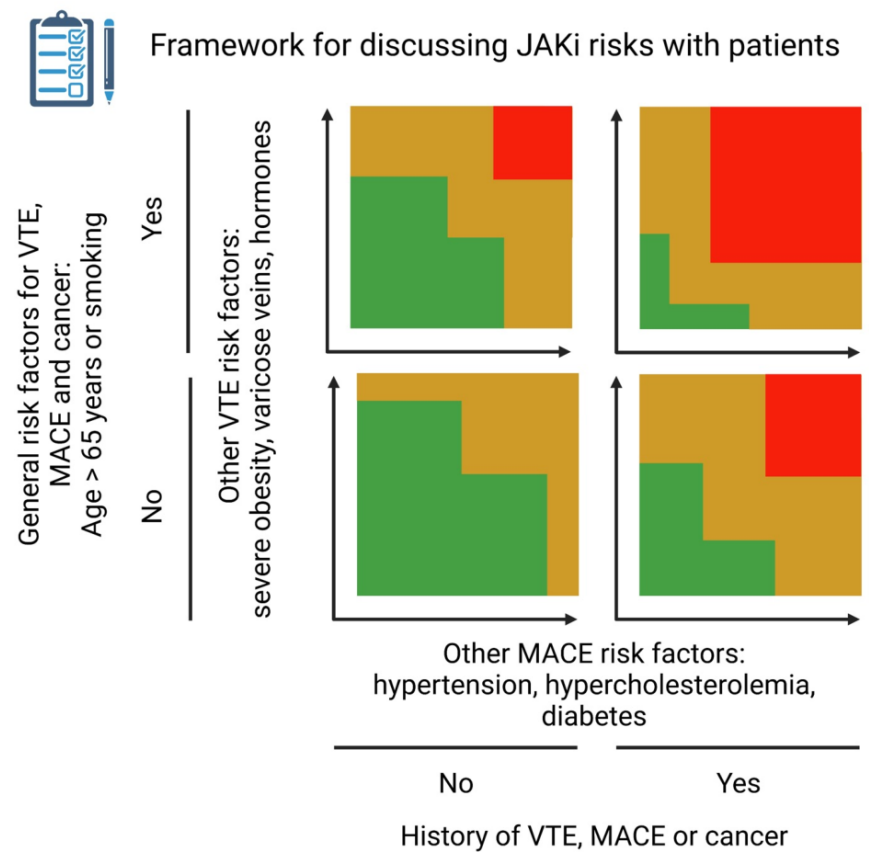

Figure 1 Framework for discussing potential JAK inhibitor risks with patients. First, identify general risk factors for VTE, MACE and cancer (age $>65$ years and smoking) and whether there is a previous history of VTE, MACE or cancer. Then, evaluate risk based on the number of other risk factors for VTE and the number of other risk factors for MACE. This is not a validated approach but a suggestion based on current (lack of) evidence and is inspired by risk charts used in cardiology. ${ }^{27}$ JAK, Janus kinase; MACE, major adverse cardiac event; VTE, venous thromboembolic event. Created with BioRender.com.

inhibitors: risk assessment, open questions about agent selection within class, but ultimately prescribing where appropriate following risk justification. To pretend such a strategy to be without precedent would be to ignore reality, and for a specialty familiar with managing uncertainty, we should not let such a problem paralyse us. Of course, these considerations may be an oversimplification. Cancer risk is harder to accurately elicit, but such an assessment would include previous history or family history of cancer (beside the general considerations about age and smoking). ${ }^{24}$

\section{WHILE WAITING FOR MORE DATA}

The current lack of broad post-marketing safety data for JAK inhibitors makes risk-benefit ratio estimations challenging for clinicians, but greater uncertainty exists in rheumatology with less controversy. ${ }^{25}$ Ultimately, 'treat-to-target' will in the end always be 'treat-to-agreement': any treatment of a patient must be based on shared decision-making. ${ }^{26} \mathrm{JAK}$ inhibitor therapy should not be an exception, even if optimal counselling might take clinicians some time to master. Therefore, rheumatologists should not be waiting for Godot. Unlike the characters in the play, who wait so long that they plan their suicide to escape their predicament, rheumatologists will have to say, "let us do something, it is safer". As we have done 
in the past, and will do in the future, the optimal treatment strategy will have to be tailored based on individual patient risk factors and preferences in a shared-decision process.

\section{Author affiliations}

${ }^{1}$ Department of Biomedicine, Aarhus University, Aarhus, Denmark

${ }^{2}$ Diagnostic Center, Silkeborg Regional Hospital, Silkeborg, Denmark

${ }^{3}$ Department of Clinical Medicine, University of Copenhagen, Kobenhavn, Denmark ${ }^{4}$ DANBIO and Copenhagen Center for Arthritis Research (COPECARE), Center for Rheumatology and Spine Diseases, Centre of Head and Orthopedics, University Hospital of Copenhagen Rigshospitalet, Glostrup, Denmark

${ }^{5}$ Department of Rheumatology, Center for Rheumatology and Spine Diseases, Centre of Head and Orthopedics, University Hospital of Copenhagen Rigshospitalet, Glostrup, Denmark

${ }^{6}$ Department of Rheumatology, Austin Health, Heidelberg, Victoria, Australia ${ }^{7}$ Department of Clinical Pharmacology and Therapeutics, Austin Health, Heidelberg, Victoria, Australia

${ }^{8}$ The Centre for Digital Transformation of Health, University of Melbourne, Parkville, Victoria, Australia

${ }^{9}$ Faculty of Medicine, University of Queensland School of Clinical Medicine, Brisbane, Queensland, Australia

${ }^{10}$ Department of Rheumatology, Royal Brisbane and Women's Hospital, Herston, Queensland, Australia

${ }^{11}$ Department of Rheumatology, Aarhus University Hospital, Aarhus, Denmark

${ }^{12}$ Department of Medicine, University of Melbourne, Parkville, Victoria, Australia

Twitter Tue Wenzel Kragstrup @KragstrupTW, Christopher McMaster @ DrCMcMaster, Philip C Robinson @philipcrobinson and David FL Liew @drdavidliew

Contributors TWK and DFLL established the project. All authors were involved in the conception and scope of the viewpoint. TWK made the first draft of the manuscript. BG, ALS, CM, PCR, BD and DFLL critically revised the manuscript. All authors approved the final version of the manuscript and agree to be accountable for all aspects of the work.

Funding The authors have not declared a specific grant for this research from any funding agency in the public, commercial or not-for-profit sectors.

Competing interests TWK: Speaking fees from Pfizer, Bristol-Myers Squibb, Eli Lilly, Novartis, UCB and AbbVie. Consultancy fees from Bristol-Myers Squibb and Gilead. Research grants from Gilead. Co-founder and clinical developer in iBio Tech ApS. BG: Research grants from Pfizer, BMS, AbbVie. BD: Speaking fees from Pfizer, Novartis and AbbVie. Consultancy fees from Boehringer Ingelheim, AstraZeneca, AbbVie, Novartis, Gilead, Eli Lilly. Research grants: Gilead and AbbVie. PCR reports personal fees from AbbVie, Atom Biosciences, Eli Lilly, Gilead, GlaxoSmithKline, Janssen, Kukdong, Novartis, UCB, Roche, Pfizer; meeting attendance support from BMS, Pfizer and UCB; and grant funding from Janssen, Novartis, Pfizer and UCB Pharma.

Patient consent for publication Not applicable.

Provenance and peer review Not commissioned; externally peer reviewed.

Open access This is an open access article distributed in accordance with the Creative Commons Attribution Non Commercial (CC BY-NC 4.0) license, which permits others to distribute, remix, adapt, build upon this work non-commercially, and license their derivative works on different terms, provided the original work is properly cited, appropriate credit is given, any changes made indicated, and the use is non-commercial. See: http://creativecommons.org/licenses/by-nc/4.0/.

\section{ORCID iDs}

Tue Wenzel Kragstrup http://orcid.org/0000-0002-6439-397X

Bente Glintborg http://orcid.org/0000-0002-8931-8482

Philip C Robinson http://orcid.org/0000-0002-3156-3418

Bent Deleuran http://orcid.org/0000-0002-7079-1587

David FL Liew http://orcid.org/0000-0001-8451-8883

\section{REFERENCES}

1 Administration UFaD. FDA requires warnings about increased risk of serious heart-related events, cancer, blood clots, and death for JAK inhibitors that treat certain chronic inflammatory conditions, 2021.

2 Agency EM. Xeljanz (tofacitinib) summary of product characteristics, 2021. Available: https://www.ema.europa.eu/en/documents/productinformation/xeljanz-epar-product-information_en.pdf [Accessed 2 Feb 2022].
3 Administration USFD. Xeljanz (tofacitinib) highlights of prescribing information, 2021. Available: https://www.accessdata.fda. gov/drugsatfda_docs/label/2021/203214s028,208246s013, 213082s003lbl.pdf [Accessed 2 Feb 2022].

4 Harigai M, Honda S. Selectivity of Janus kinase inhibitors in rheumatoid arthritis and other immune-mediated inflammatory diseases: is expectation the root of all headache? Drugs 2020;80:1183-201.

5 Nash P, Kerschbaumer A, Dörner T, et al. Points to consider for the treatment of immune-mediated inflammatory diseases with Janus kinase inhibitors: a consensus statement. Ann Rheum Dis 2021;80:71-87.

6 Administration UFaD. FDA requires warnings about increased risk of serious heart-related events, cancer, blood clots, and death for JAK inhibitors that treat certain chronic inflammatory conditions 12/2021 update, 2021.

7 Charles-Schoeman C, Greenwald M, Wang C. The Risk of Venous Thromboembolic Events in Patients with RA Aged $\geq 50$ Years with $\geq 1$ Cardiovascular Risk Factor: Results from a Phase 3b/4 Randomized Safety Study of Tofacitinib vs TNF Inhibitors [abstract]. Arthritis Rheumatol 2021;73.

8 Charles-Schoeman C, Bhatt D, Giles J, et al. Risk Factors for Major Adverse Cardiovascular Events in Patients Aged $\geq 50$ Years with RA and $\geq 1$ Additional Cardiovascular Risk Factor: Results from a Phase 3b/4 Randomized Safety Study of Tofacitinib vs TNF Inhibitors [abstract]. Arthritis Rheumatol 2021;73.

9 Curtis J, Gunay L, Sugiyama N, et al. Malignancies in Patients Aged $\geq 50$ Years with RA and $\geq 1$ Additional Cardiovascular Risk Factor: Results from a Phase 3b/4 Randomized Safety Study of Tofacitinib vs TNF Inhibitors [abstract]. Arthritis Rheumatol $2021 ; 73$.

10 Ytterberg SR, Bhatt DL, Mikuls TR, et al. Cardiovascular and cancer risk with tofacitinib in rheumatoid arthritis. $N$ Engl J Med 2022;386:316-26.

11 Pfizer. Pfizer announces modification to ongoing tofacitinib FDA post-marketing requirement study in patients with rheumatoid arthritis, 2019.

12 Charles-Schoeman CHC, Guan S, Parikh N. Relationship Between Paraoxonase-1 Genotype, Activity, and Malignancies in Patients with RA Receiving Tofacitinib [abstract]. Arthritis Rheumatol 2021;73.

13 Cohen SB, Tanaka Y, Mariette X, et al. Long-Term safety of tofacitinib up to 9.5 years: a comprehensive integrated analysis of the rheumatoid arthritis clinical development programme. RMD Open 2020;6:e001395.

14 Cohen SB, van Vollenhoven RF, Winthrop KL, et al. Safety profile of upadacitinib in rheumatoid arthritis: integrated analysis from the select phase III clinical programme. Ann Rheum Dis 2020. doi:10.1093/rheumatology/keaa111.204. [Epub ahead of print: 28 Oct 2020].

15 Smolen JS, Genovese MC, Takeuchi T, et al. Safety profile of Baricitinib in patients with active rheumatoid arthritis with over 2 years median time in treatment. $J$ Rheumatol 2019;46:7-18.

16 Winthrop KL, Tanaka Y, Takeuchi T, et al. Integrated safety analysis of filgotinib in patients with moderately to severely active rheumatoid arthritis receiving treatment over a median of 1.6 years. Ann Rheum Dis 2022;81:184-92.

17 Pharmacovigilance ENoCfPa. Available: https://www.encepp.eu [Accessed 2 Feb 2022].

18 Millett ERC, Peters SAE, Woodward M. Sex differences in risk factors for myocardial infarction: cohort study of UK Biobank participants. BMJ 2018;363:k4247.

19 Molander V, Bower H, Frisell T, et al. Risk of venous thromboembolism in rheumatoid arthritis, and its association with disease activity: a nationwide cohort study from Sweden. Ann Rheum Dis 2021;80:169-75.

20 Solomon DH, Reed GW, Kremer JM, et al. Disease activity in rheumatoid arthritis and the risk of cardiovascular events. Arthritis Rheumatol 2015;67:1449-55.

21 Strangfeld A, Hierse F, Rau R, et al. Risk of incident or recurrent malignancies among patients with rheumatoid arthritis exposed to biologic therapy in the German biologics register rabbit. Arthritis Res Ther 2010;12:R5.

22 He M, Pawar A, Desai RJ, et al. Risk of venous thromboembolism associated with methotrexate versus hydroxychloroquine for rheumatoid arthritis: a propensity score-matched cohort study. Semin Arthritis Rheum 2021;51:1242-50.

23 Khosrow-Khavar F, Kim SC, Lee H, et al. Tofacitinib and risk of cardiovascular outcomes: results from the safety of tofacitinib in routine care patients with rheumatoid arthritis (STAR-RA) study. Ann Rheum Dis 2022. doi:10.1136/annrheumdis-2021-221915. [Epub ahead of print: 13 Jan 2022]. 
24 Chatzidionysiou K, Delcoigne B, Frisell T, et al. How do we use biologics in rheumatoid arthritis patients with a history of malignancy? An assessment of treatment patterns using Scandinavian registers. RMD Open 2020;6:e001363.

25 George MD, Baker JF, Winthrop K, et al. Risk for Serious Infection With Low-Dose Glucocorticoids in Patients With Rheumatoid Arthritis : A Cohort Study. Ann Intern Med 2020;173:870-8.
26 Nikiphorou E, Santos EJF, Marques A, et al. 2021 EULAR recommendations for the implementation of self-management strategies in patients with inflammatory arthritis. Ann Rheum Dis 2021;80:1278-85.

27 Ueda P, Woodward M, Lu Y, et al. Laboratory-Based and office-based risk scores and charts to predict 10-year risk of cardiovascular disease in 182 countries: a pooled analysis of prospective cohorts and health surveys. Lancet Diabetes Endocrinol 2017;5:196-213. 